\title{
Evolución de la caries en los escolares incluidos en la población diana de un programa público de salud bucodental en dos zonas básicas de salud de Castilla-La Mancha
}

\author{
Navarro Montes I*, Peso de Ojeda L*, Represa González J**, González Sanz A****
}

\section{RESUMEN}

En el presente trabajo se describen las actuaciones preventivas que desde el ámbito de actuación de la Atención Primaria, se desarrollan en el marco asistencial de la sanidad pública en las Zonas Básicas de Salud de Castilla La Mancha. La población diana que se beneficia de las acciones preventivas que contempla el Programa de Salud Bucodental (PSB) de esta Comunidad Autónoma, la constituyeron en este trabajo 2749 escolares, que desde el año 1994 hasta el curso académico 1999-2000 pertenecieron a cuarto curso de primaria. Hemos podido concluir tras el estudio, que los escolares que participaron más fielmente en las actividades del PSB presentaban ya, antes de su inclusión en dicho programa, una mejor salud bucodental que aquellos que no acudieron al mismo. También constatamos en este estudio el relevante descenso del índice CAOD a lo largo de los años del mismo y el irrelevante índice de restauración (I.R) hallado. Por último analizamos la relación existente entre el nivel de implantación social del PSB, a lo largo del tiempo con el incremento en la captación y fidelización de los escolares participantes en él.

Palabras clave: Caries dental, prevalencia de caries, índices de caries.

\section{SUMMARY}

In the present work the preventive performances are described that from the ambit of performance of Primary Attention, are developed in the frame of the health publishes in the Basic Zones of Health of Castilla La Mancha. The population that benefits from the actions for provisional remedy that contemplates to the Bucodental Health Program (BHP) of this Autonoma Community, constituted in this work 2749 students, who from year 1994 to academic course 1999-2000 belonged to fourth course of primary. We have been able to conclude after the study, that the students who participated faithfully more in the activities of the PBH already presented, before their inclusion in this program one better bucodental health than those that they did not go to the same one. Also we stated in this study the excellent reducction of the DMFT index throught the years of same and irrelevant index of restoration (FT/DMFT) found. Finally we will analyze the existing relation between the social level of implantation of BHP, through the time with the increase in the pick up and fidelity of the participant students in this program.

Key words: Dental caries, caries prevalence, caries index.

* $\quad$ Odontólogo de área del INSALUD de Ciudad Real.

** Especialista Universitario en Salud Pública Oral.

*** Profesor Titular Dpto. Estomatología IV. Fac. Odontología U.C.M.

Navarro Montes I, Peso de Ojeda L, Represa González J, González Sanz A. Evolución de la caries en los escolares incluidos en la población diana de un programa público de salud bucodental en dos zonas básicas de salud de Castilla-La Mancha. Av. Odontoestomatol 2003; 19-1: 35-42 


\section{INTRODUCCIÓN}

Desde 1.992 la provincia de Ciudad Real cuenta con USB (Unidades de Salud Bucodental) ubicadas dentro de los EAP (Equipos de Atención Primaria), en ellas se desarrolla el PSB (Programa de Salud Bucodental) contemplado en el catálogo de prestaciones del Ministerio de Sanidad del año $1995{ }^{(1)}$.

El punto de partida es la encuesta epidemiológica realizada en Catilla La Mancha (2), donde ya se evidenciaban niveles más bajos de caries en comparación con otras Comunidades Autónomas.

Con la puesta en marcha de los programas de atención dental en el territorio español, la prevalencia de caries ha disminuido a lo largo de los años ${ }^{(3)}$. Con la instauración del PADI (Programa de Atención Dental Infantil), el Pais Vasco ha visto disminuir su índice CAOD con el paso del tiempo ${ }^{(4)}$.

En los países industrializados europeos, el descenso de la prevalencia de caries se observó mucho antes que en España; también es cierto que los programas preventivos se pusieron en marcha antes que en el nuestro ${ }^{(5)}$.

Otros factores han sido relacionados con la reducción de caries en la población. Así se ha publicado que la relación de estos descensos, puede deberse al mayor contacto de la superficie dental con el flúor (agua fluorada, suplementos de flüoruro y otros productos fluorados), además de una mayor concienciación social ante la salud dental ${ }^{(6)}$, que ha repercutido en la modificación de determinados hábitos alimenticios de marcado efecto cariogénico.

Durante el desarrollo natural de una enfermedad se pueden realizar diferentes tipos de prevención. En la asistencia primaria odontológica se puede actuar sobre el periodo prepatogénico, realizándose una prevención primaria o bien en una fase temprana de la patología mitigando los factores de riesgo y así realizándose una prevención secundaria.

Las medidas preventivas llevadas a cabo en los PSB, normalmente las constituyen la Educación para la Salud Bucodental (ESB), instrucción a la higiene dental (técnicas de cepillado y uso de seda dental),

\begin{tabular}{|c|c|c|c|}
\hline \multirow[t]{2}{*}{ Edad } & 9 & 2.105 & $(76,57 \%)$ \\
\hline & 10 & 644 & $(23,43 \%)$ \\
\hline \multicolumn{2}{|l|}{ TOTAL } & 2.749 & \\
\hline \multirow[t]{2}{*}{ Sexo } & V & 1.372 & $(49,90 \%)$ \\
\hline & $M$ & 1.377 & $(50,10 \%)$ \\
\hline \multicolumn{2}{|l|}{ TOTAL } & 2.749 & \\
\hline
\end{tabular}

modificación de hábitos dietéticos, control de placa bacteriana y aplicación de flúor y selladores de fisuras en el primer molar permanente según Normas Técnicas Mínimas (NTM).

También se realiza en estos programas odontología conservadora como es la obturación del molar de los seis años, según la cartera de servicios del sistema de salud.

\section{OBJETIVOS}

Nos planteamos los siguientes objetivos, en el presente trabajo:

1) Conocer las diferentes fases evolutivas que la caries dental ha seguido a lo largo de los seis años de nuestro estudio.

2) Analizar si la receptividad al PSB por parte de la población diana está relacionada o no con su nivel previo de salud bucodental.

\section{MATERIAL Y MÉTODO}

Desde el año 1994 hasta el curso académico 19992000 estudiamos un total de 2.749 escolares de 9 y 10 años de edad, de los cuales 1.372 eran varones y 1.377 niñas, que pertenecían a seis poblaciones de la provincia de Ciudad Real. Al comienzo del estudio todos ellos cursaban cuarto de primaria. El tamaño muestral se corresponde con la totalidad de la población diana. En la Tabla 1 se describen las características de nuestra muestra, como son la edad y el sexo. 


\section{TABLA 2.- DESCRIPCIÓN DE LA VARIABLE TRATAMIENTO. EXPLORACIÓN INICIAL}

\begin{tabular}{|cccr|}
\hline & Tratados & \multicolumn{1}{c}{ No tratados } & \multicolumn{1}{c|}{ Total } \\
\hline 1994 & $328(72,72 \%)$ & $123(27,28 \%)$ & 451 \\
1995 & $298(72,33)$ & $114(27,67 \%)$ & 412 \\
1996 & $304(70,37 \%)$ & $128(29,63 \%)$ & 432 \\
1997 & $386(71,21 \%)$ & $156(28,79 \%)$ & 542 \\
1998 & $405(92,46 \%)$ & $33(7,54 \%)$ & 438 \\
1999 & $474(100 \%)$ & 0 & 474 \\
Total & $2.195(79,84 \%)$ & $554(20,16 \%)$ & 2.749 \\
\hline
\end{tabular}

De los 2.749 niños 2.195 siguieron de forma voluntaria un PSB y 554 no lo hicieron. A lo largo de este trabajo denominaremos a los primeros como niños tratados y a los segundos como no tratados; este aspecto queda reflejado en la Tabla 2.

El ESB (Equipo de Salud Bucodental) que realizó el "trabajo de campo", estaba constituido por una subunidad de salud bucodental (un odontólogo y una auxiliar de clínica).

En una primera visita todos los niños fueron vistos en el colegio, disponiendo de una ficha-historia para cada uno de ellos, en la que quedaron reflejados los datos de filiación y el odontograma. En éste se recogían, entre otros datos de interés, los siguientes: información de todos los dientes temporales y permanentes (dientes presentes y ausentes, caries, obturaciones y pérdida por caries).

La exploración se efectuó en el aula a la que pertenecían los niños con luz natural, espejos y sondas, utilizando éstas tan sólo cuando el diagnóstico visual ofrecía dudas ${ }^{(7)}$. Preferimos realizar la exploración antes de la hora del recreo.

Finalizada la exploración se le entregaba a cada escolar una carta dirigida a los padres, donde se les explicaba las características del programa, invitándoles a participar con su hijo en el mismo.

Características del PSB. El programa se llevó a cabo en el ámbito de la Atención Primaria en las USB de los dos centros de salud en los que trabajamos. Contempla medidas preventivas, interceptivas y ope- rativas. Entre las preventivas destacamos la instrucción al cepillado, uso de seda dental, control de placa y de dieta, aplicación de flúor tópico y selladores de fosas y fisuras en el primer molar permanente. Entre las interceptivas destaca la corrección de hábitos y entre las operativas la obturación del molar de los seis años.

La primera cita se realizó en la sala de educación del centro, en la cual se presentaba el ESB, el programa y se proyectaba una película educativa. Posteriormente pasábamos al gabinete dental, donde se confirmaba el odontograma codificado en el colegio.

Las siguientes citas dependían de las necesidades de tratamiento (selladores y/u obturaciones). Una vez terminado este protocolo, estaban contempladas en el programa las revisiones semestrales hasta que el niño tuviera catorce años de edad. En función de la asistencia o no asistencia de los escolares a estas revisiones programadas, se clasificaron éstas como esporádicas, periódicas y estrictas.

Diseño metodológico. Realizamos un estudio transversal correspondiente a los 9-10 años de edad y se analizó longitudinalmente a la población diana desde 1994 hasta el curso académico 1999-2000.

Se estudió el índice CAO descrito por Klein, Palmer y Knutson en 1.938 (8), considerando la $C$ como dientes cariados, la $A$ como ausentes por caries y la $O$ como obturados. El CAOM es el índice CAO pero referido al primer molar permanente. La medida de prevalencia homónima utilizada para la dentición primaria ha sido el cod, no teniendo en cuenta aquí los dientes deciduales perdidos. Al realizar el I.R (Indice de restauración) dividimos el número de dientes obturados por el CAOD o el cod según sea dentición permanente o decidual.

Método estadístico.El estudio de la concordancia que describen Landis y Koch (9) lo realizamos en una fase previa antes de empezar el programa y después durante el trabajo de campo.

Las variables del estudio se clasificaron en Cualitativas o Categóricas en escala nominal y ordinal y variables Cuantitativas. 


\begin{tabular}{|ccc|}
\hline & \multicolumn{2}{c|}{$\begin{array}{l}\text { TABLA 3.- ÍNDICE COD. } \\
\text { EXPLORACIÓN INICIAL }\end{array}$} \\
\hline & Tratados & No tratados \\
\hline 1994 & 1,55 & 1,67 \\
1995 & 1,15 & 2,63 \\
1996 & 0,62 & 2,32 \\
1997 & 1,34 & 2,67 \\
1998 & 1,24 & 2,03 \\
1999 & 1,27 & \\
\hline
\end{tabular}

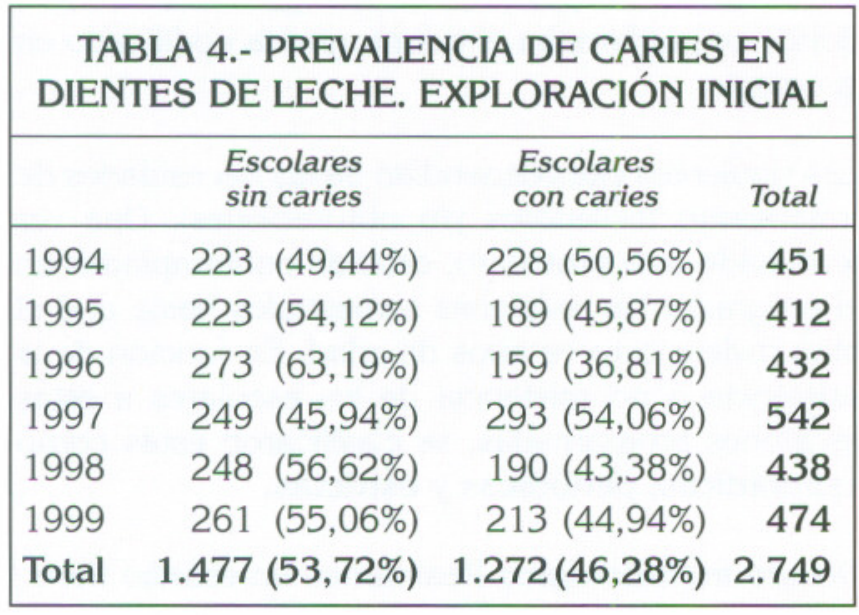

En la estadística descriptiva utilizamos medidas de centralización (media aritmética y moda), de dispersión (desviación típica) y de forma.

En la estadística inferencial se utilizó la prueba del JiCuadrado y medidas de asociación, escala de medida nominal y escala de medida ordinal o intervalo.

\section{RESULTADOS}

Obtuvimos en el año 1994 una concordancia del $88,7 \%$ y en 1997 la concordancia obtenida fue del 93,4\%; concordancia "casi completa" según Landis y Koch ${ }^{(9)}$ en las dos ocasiones.

El índice cod en el año 1994 de los niños no tratados fue de 1,67 mientras que en los escolares denominados como tratados fue de 1,55. Todos los datos de este índice durante los años del estudio se expresan en la Tabla 3.

\begin{tabular}{|c|c|c|}
\hline \multicolumn{3}{|c|}{$\begin{array}{l}\text { TABLA 5.- ÍNDICE CAOD. } \\
\text { EXPLORACIÓN INICIAL }\end{array}$} \\
\hline & Tratados & No tratados \\
\hline 1994 & 2,29 & 2,36 \\
\hline 1995 & 2,23 & 2,04 \\
\hline 1996 & 2,17 & 2,52 \\
\hline 1997 & 1,72 & 1,95 \\
\hline 1998 & 0,94 & 1,42 \\
\hline 1999 & 0,51 & \\
\hline
\end{tabular}

\begin{tabular}{|c|c|c|c|c|}
\hline \multicolumn{5}{|c|}{$\begin{array}{l}\text { TABLA 6.- PREVALENCIA DE CARIES EN } \\
\text { DIENTES DEFINITIVOS. EXPLORACIÓN INICIAL }\end{array}$} \\
\hline & $\begin{array}{l}\text { Escolares } \\
\text { sin caries }\end{array}$ & & $\begin{array}{l}\text { Escolares } \\
\text { on caries }\end{array}$ & Total \\
\hline 1994 & $152(33,70 \%)$ & 299 & $(66,30 \%)$ & 451 \\
\hline 1995 & $141(34,22 \%)$ & 271 & $(65,78 \%)$ & 412 \\
\hline 1996 & $136(31,48 \%)$ & 296 & $(68,52 \%)$ & 432 \\
\hline 1997 & $190(35,05 \%)$ & 352 & $(64,95 \%)$ & 542 \\
\hline 1998 & $296(67,57 \%)$ & 142 & $(32,43 \%)$ & 438 \\
\hline 1999 & $386(81,43 \%)$ & 88 & $(18,57 \%)$ & 474 \\
\hline Total & $1.301(47,32 \%)$ & 144 & $(52,68 \%)$ & 2.749 \\
\hline
\end{tabular}

Referente a la prevalencia de caries en dentición decidual los resultados obtenidos desde 1994 a 1999 quedan reflejados en la Tabla 4.

El índice CAOD también referente a la exploración inicial que se realizó durante el estudio a todos los escolares lo exponemos en la Tabla 5. El CAOD de los niños tratados de 1994 fue de 2,29 y el de los no tratados de 2,36. Para este mismo índice y para estos mismos grupos en 1998 las cifras arrojadas fueron de 0,94 y 1,42 respectivamente.

La prevalencia de caries en dientes definitivos se expresa en la Tabla 6; también nos referimos aquí a la exploración inicial realizada desde 1994 a 1999.

El CAOM se presenta en la Tabla 7, hacemos distinción en esta tabla como en las anteriores entre los niños según la variable tratados y no tratados. En 1997 este índice en los tratados era de 1,67 y de 1,95 en los no tratados. 


\begin{tabular}{|c|c|c|}
\hline \multicolumn{3}{|c|}{$\begin{array}{l}\text { TABLA 7.- ÍNDICE CAOM. } \\
\text { EXPLORACIÓN INICIAL }\end{array}$} \\
\hline & Tratados & No tratados \\
\hline 1994 & 2,26 & 2,33 \\
\hline 1995 & 2,16 & 2,04 \\
\hline 1996 & 2,16 & 2,52 \\
\hline 1997 & 1,67 & 1,95 \\
\hline 1998 & 0,91 & 1,42 \\
\hline 1999 & 0,51 & \\
\hline
\end{tabular}

TABLA 8.- ÍNDICE DE RESTAURACIÓN EN DIENTES DEFINITIVOS. EXPLORACIÓN INICIAL

\begin{tabular}{|lll|}
\hline & No tratados & Tratados \\
\hline $1^{\circ}$ & 0.1 & 0.06 \\
$2^{\circ}$ & 0.06 & 0.07 \\
$3^{\circ}$ & 0 & 0.1 \\
$4^{\circ}$ & 0 & 0.05 \\
$5^{\circ}$ & 0.03 & 0.08 \\
$6^{\circ}$ & & 0.1 \\
\hline
\end{tabular}

\begin{tabular}{|lrrrrrrr|}
\hline \multicolumn{7}{|c|}{ TABLA 9.- FRECUENCIA DE REVISIONES } \\
\hline Año & Esporádica & $\%$ & Periódica & \multicolumn{1}{c|}{$\%$} & Estricta & $\%$ & Total \\
\hline $1^{\circ}$ & 81 & 24,69 & 147 & 44,82 & 100 & 30,49 & 328 \\
$2^{\circ}$ & 91 & 30,54 & 120 & 40,26 & 87 & 29,20 & 298 \\
$3^{\circ}$ & 98 & 32,24 & 99 & 32,56 & 107 & 35,20 & 304 \\
$4^{\circ}$ & 68 & 17,60 & 87 & 22,54 & 231 & 59,86 & 386 \\
$5^{\circ}$ & 0 & & 0 & & 405 & 100 & 405 \\
Total & 338 & $\mathbf{1 9 , 6 3}$ & 453 & $\mathbf{2 6 , 3 2}$ & 930 & $\mathbf{5 4 , 0 5}$ & $\mathbf{1 7 2 1}$ \\
\hline
\end{tabular}

El Índice de Restauración en dentición decidual fue de 0,015 para los niños no tratados y para la totalidad de los años del estudio; los niños tratados presentaron valores de este índice de 0,03.

En la Tabla 8 expresamos el Índice de Restauración en dentición permanente. En 1997 y 1998, en el grupo de los no tratados este índice fue de 0 .

En cuanto a la calidad en la asistencia de los escolares al programa, los resultados obtenidos los describimos en la Tabla 9. El porcentaje de revisiones estrictas en 1998 fue del 59,86\%.

\section{DISCUSIÓN}

En la literatura existen pocos estudios que se hayan realizado con muestras de la misma edad que la nuestra (9-10 años), ya que la OMS-FDI ${ }^{(10)}$ no las ha considerado como edades índice (6-12-15 años).

Refiriéndonos a la experiencia de caries en dentición decidual coincidimos con Heller et al ${ }^{(11)}$, en una de sus conclusiones tras realizar un trabajo en América del Norte en 1994; decía que había un estancamiento en la experiencia de caries en dentición primaria. Comentaba este autor que la protección del flúor en esta dentición había llegado a su máximo nivel. Manau y Cuenca ${ }^{(12)}$ sin embargo vieron como el flúor había provocado una reducción significativa de los índices de caries en dientes primarios, aunque también había aumentado el número de niños libres de caries en dentición permanente.

Los valores de nuestros índices cod se asemejan a los que encontraron Cortés et al ${ }^{(13)}$ en Navarra en $1998^{(1,29)}$. Poveda ${ }^{(14)}$ en Cuenca a los seis años de edad encontró un cod de 1,36.

La prevalencia de caries en dentición decidual de nuestra población diana, ha sido inferior a la encontrada por otros autores en muestras de edad similar a la nuestra ${ }^{(15,16)}$.

El índice CAOD ha disminuido a lo largo de los años del estudio, lo mismo ha ocurrido con la prevalencia de caries en dientes definitivos. Nuestra población diana ha experimentado este descenso de forma 
más tardía que el que experimentaron Australia, Nueva Zelanda ${ }^{(17)}$ y la Unión Europea en la década de los $70^{(18,19)}$. Como en nuestra población también en Grecia ${ }^{(20)}$ la caries empezó a disminuir de forma tardía.

El notable descenso puede ser explicado por un lado por la tendencia descentente de los últimos años en nuestro país y en otros países ${ }^{(21,22)}$, por la incorporación de medidas preventivas y mayor concienciación de la población ${ }^{(23)}$. La combinación de todas estas medidas potenciaría el efecto de una sola, dándose así un mecanismo sinérgico ${ }^{(24)}$.

También como el cod nuestro CAOD se asemeja al encontrado por Cortés et al en su estudio ${ }^{(13)}$.

Francia ha presentado los valores más elevados de la prevalencia de caries en dentición permanente, según expusieron Cahen et al ${ }^{(24)}$ en su trabajo.

Hemos encontrado valores semejantes del índice CAOD y del índice CAOM, quizás algo inferiores en este último; esto lo explica la edad de la muestra. Rosado et al ${ }^{(26)}$, también coinciden con nosotros en esta afirmación.

El I.R lo hemos catalogado en nuestro estudio como muy bajo. Cuenca et al ${ }^{(27)}$ decían que la proporción de caries que se obturaba era irrelevante, lo que dejaba entrever las altas necesidades de tratamiento que existían y el bajo nivel de atención de dichas necesidades. Aunque nos hemos encontrado cifras de este índice en la literatura muy bajas ${ }^{(28)}$, en Guitiriz en 1999 se presentaron para niños de 10 años de edad cifras del I.R del $62 \%{ }^{(29)}$, bastante aceptables por cierto.

Hemos podido observar como los índices de caries han sido inferiores en la población, en el grupo de los niños tratados que en el de los no tratados. Pankala et al ${ }^{(30)}$, también comentaban en su trabajo que los sujetos que abandonaron el programa después de su examen inicial, tenían una incidencia estadísticamente más significativa de nuevas caries que aquellos que habían participado durante todo el estudio. Aquellos con salud oral más deficiente abandonaron más fácilmente el sistema de cuidados sanitarios.
Hemos constatado al igual que Imfeld (31), que la demanda de la atención odontológica se ha incrementado notablemente en el transcurso de los años, sin duda este aspecto ayuda a disminuir el número de caries por sujeto.

\section{CONCLUSIONES}

A la luz de los resultados obtenidos hemos podido concluir los siguientes puntos:

1) El cod de la muestra no ha experimentado modificación relevante durante todos los años de estudio.

2) La prevalencia de caries en dentición decidual ha seguido el mismo patrón evolutivo que el cod.

3) El índice CAOD ha experimentado un notable descenso.

4) La prevalencia de caries en dentición permanente al igual que el índice CAOD, ha presentado un relevante descenso a lo largo de los años del estudio.

5) Debido a la edad de la muestra, el CAOD y el CAOM han experimentado una evolución similar.

6) El Índice de Restauración ha sido irrelevante.

7) Los niños que voluntariamente decidieron acudir al programa, presentaron índices y prevalencia de caries iniciales inferiores que aquellos que no lo hicieron.

8) Conforme se ha ido implantando el programa a lo largo de los años, ha producido impacto social, aumentando así el número de niños que asistieron de forma estricta y regular al mismo.

\section{BIBLIOGRAFÍA}

1. BOE. Prestaciones sanitarias facilitadas directamente a las personas por el Sistema Nacional de Salud y financiadas con cargo a la Seguridad Social o fondos estatales adscritos a la sanidad. 10 Febrero 1995. Anexo I. P. 4551.

2. Fernández I, Sánchez F, Domínguez JA, Barbero JC, Ráez E, Peso L. Consejería de Sanidad. Encuesta de Salud Bucodental Infantil en Castilla 
La Mancha. Dirección General de Salud Pública; 1994. Servicios de Información y Ordenación Sanitaria.

3. Cuenca E, Casals E, Martínez Lizán I, Manau C, Salleras LI. Encuesta Epidemiológica de Caries entre los escolares de Cataluña. Arch Odontoestomatol Prev Comunitaria 1997; 13 (2): 765-772.

4. Simón F. Tesis Doctoral. Evaluación de los seis primeros años de desarrollo del Programa de Asistencia Dental Infantil (PADI) de la Comunidad Autónoma de Pais Vasco. Universidad Complutense de Madrid. Facultad de Medicina, 1996.

5. Burt BA. Influences for change in the dental health status of population: An historical perspective. J- Public-Health-Dent 1978; 38: 272278.

6. Renson CE. Global Changes in Caries Prevalence and Dental Manpower Requeriments: 1. Assembling and Analyzing the Data. DentPublic-Health 1989; 16 (7): 287-298.

7. OMS. Encuesta de salud bucodental. Métodos Básicos. Cuarta edición. Ginebra, 1997.

8. Klein H, Palmer CE, Knutson JW. Studies on dental caries. Dental status and dental needs of elementary schoolchildren. U.S. Public-HealthRep 1938; 53: 751-65.

9. Landis JR, Koch GG. The measurement of observer agreement for categorical data. Biometrics 1977; 33: 159-74.

10. FDI/OMS. Patrones cambiantes de Salud Bucodental e implicaciones para los recursos humanos dentales. Parte Primera. Arch Odontoestomatol 1986; 2: 23-40.

11. Heller KE, Szpuner SM, Burt BA. Changes in children's oral Health Status from 1986 to 1993. JDent-Res 1994; 64: 103-116.

12. Manau C, Cuenca E. Resultados de la evaluación del programa de enjuagues con fluoruro sódico, realizado por los escolares de Cataluña, resumen informe preliminar. Acta Esp Estomatol 1987; 365: 51-53.

13. Cortés J, Doria A, Martínez I, Asenjo MA, Sainz de Murieta I, Cuenca E. Prevalencia de caries dental entre la población infantil de Navarra. Arch Odontoestomatol Prev Comunitaria 1998; 14 (11): 640-646.

14. Poveda R. Estudio epidemiológico transversal de caries en población rural infantil de Cuenca. Rev Actual Estomatol Esp 1989; 388: 65-73.

15. García-Camba JM, Oñorbe M, Gómez F, Blázquez $M$, Rodríguez $M V$, Serra $P$, et al. Consejería de Sanidad de Madrid. Encuesta sobre la salud buco-dental de la población infantil de la Comunidad de Madrid. Dirección General de prevención y promoción de la Salud 1992; Documentos Técnicos de Salud Pública $n^{\circ} 3$.

16. Fejerskov O, Baelum V, Luan W, Manji F. Caries prevalence in Africa and the People's Republic of China. Int-Dent-J 1994; 44: 425-433.

17. Hansen H, Milen A, Tala H, Nordling H, Paunio I, Heinonen O. Caries frecuency among 6-17years-old participants of the Finish Public Dental Care during 1975-79. Community-Dent-OralEpidemiol 1983; 11: 64-80.

18. O'Mullanen D. Eficiency in Oral Health Care. Devaluation of Oral Health Systems in Europe. Bio- Eur 1997; 16: 213-216.

19. Marthaler TM. Caries Status in Europe and Predictions of future trends. Caries-Res 1990; 24: 381-96.

20. Oulis C, Dratsa I, Vierrow A, Athanasouli T. Dental needs of school children of the district of Athens. Helenic-Stomat-Prev 1990; 34: 29-39.

21. Noguerol B, Llodra JC, Sicilia A, Follana M. La Salud Bucodental en España 1994, antecedentes y perspectivas de futuro. Madrid: Avances; 1995. 
22. Marthaler TM. The prevalence of dental caries in Europe 1990-1995. Caries-Res 1996; 17: 237-255.

23. Riobóo R. La evolución de la salud buco-dental en la población europea durante la última década: Variables implicadas. Av Odontoestomatol 1998; 14 (2): 119-131.

24. Rolla G, Ogaard B, De Al meida C. Clinical effect and mechanism of cariostatic action of fluoridencontaining tooth paste: a review. Int-Dent-J 1991; 41: 171-174.

25. Cahen PM, Turlot JC, Frank RM, Obbry-Musset AM. National survey of Caries Prevalence in 6-15 years-old children in France. J-Dent-Res 1989; 68 (1): 64-68.

26. Rosado J, González A, Gil FJ, Maroto E, Alós L. Estudio del estado de salud dental de una población de Torrejón de Ardoz: APS. Comunicación XX Congreso Nacional y VII Internacional de la Sociedad española de OdontoEstomatología Preventiva y Comunitaria; 1997 Oct 31-Nov 1-2; Zaragoza, España; 1997. p 60-61.

27. Cuenca E, Casals E, Martínez Lizán I, Manau C, Salleras Ll. Encuesta Epidemiológica de Caries entre los escolares de Cataluña. Arch Odon-toesto- matol Prev Comunitaria 1997; 13 (2): 765-772.

28. Fuentes S, Farrouh S. Estudio de la prevalencia de caries dental en niños de un zona básica de salud. Aten Primaria 1992; 10 (7): 861-864.

29. López XC, Smyth E. Estudio epidemiológico de la caries dental en la población escolar del Ayuntamiento de Guitiriz. Arch Odontoestomatol 1999; 151: 25-35.

30. Pankala K, Kentala J, Laippala P, Mattila K. Changes in the oral health of adolescents threated by the finish public dental services between the ages of 13 and 15 years. Community-DentOral-Epidemiol 1998; 26: 49-154.

31. Imfeld T, Steiner M, Menhini G, Marthaler T. Prediction of future high increments for children in a school dental service and private practice. JDent-Educ 1995; 59: 941-944.

\section{CORRESPONDENCIA}

Prof. ANGEL GONZALEZ SANZ

FACULTAD DE ODONTOLOGÍA UCM DEPARTAMENTO DE ESTOMATOLOGÍA IV prevent@odon.ucm.es 